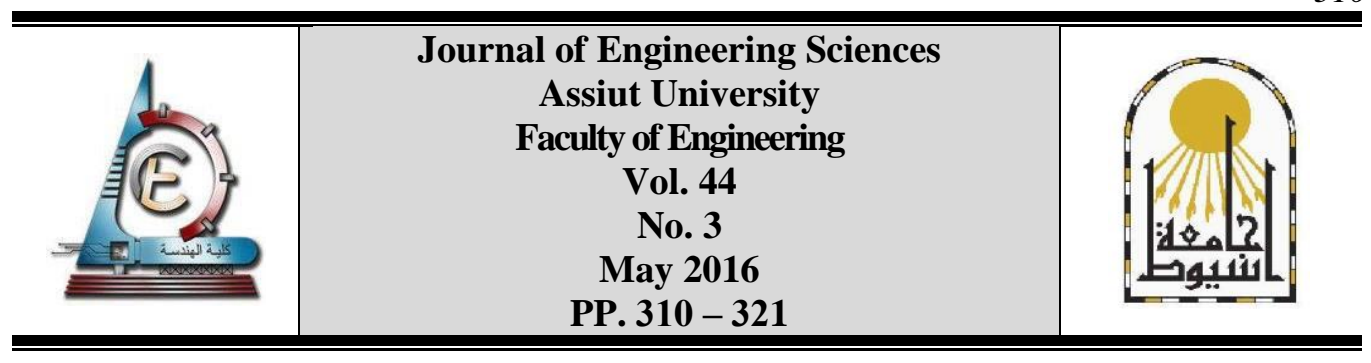

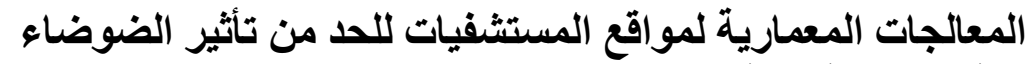

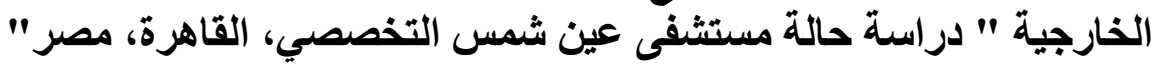

صالح يوسف الفرد"، محمد عبد الوهاب العزازى، نادي عبد الكريم مصطفى، محمد عبد السميع عيد

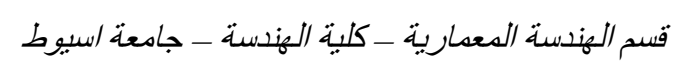

Received 18 April 2016; Accepted 12 May 2016

\section{ملخص}

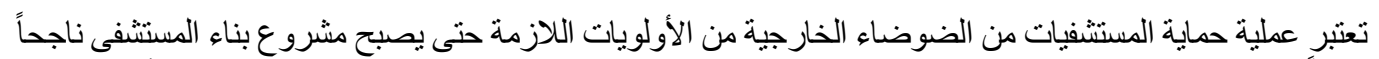

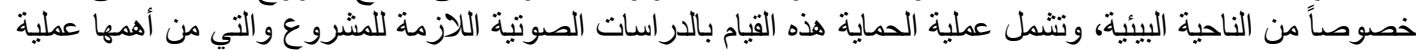

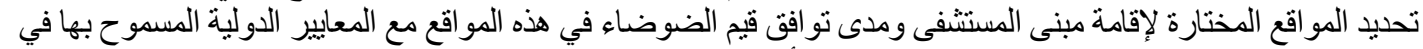

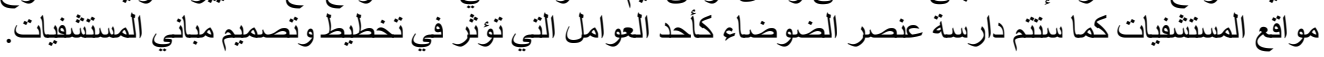
وتهدف الدراسة إلى إيجاد وسائل يمكن من خلالها الحد من الضوضاء بموقع مستشفى جامعة عين شمس

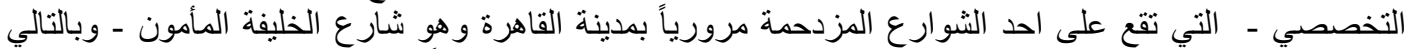

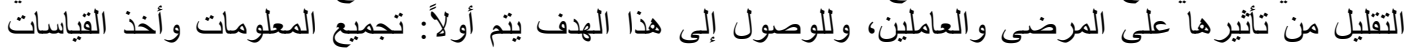

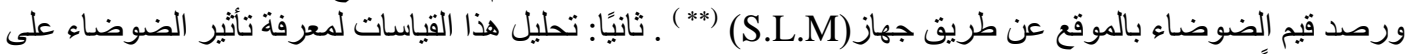

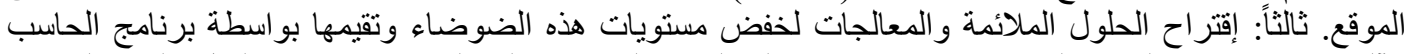

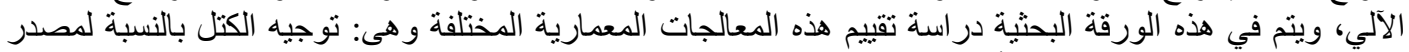

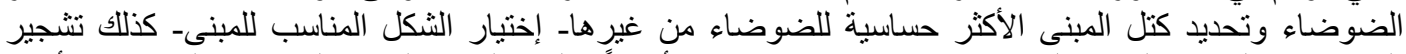

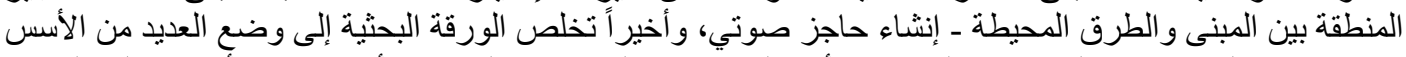

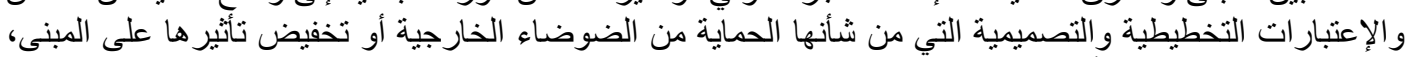

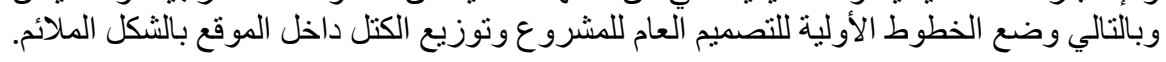

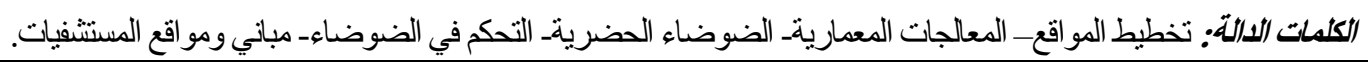

\section{1 المقدمــة}

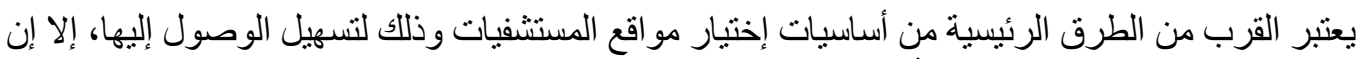

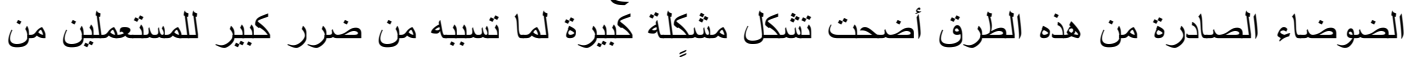

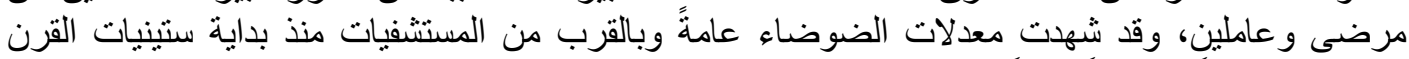

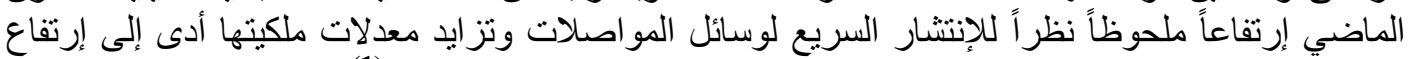

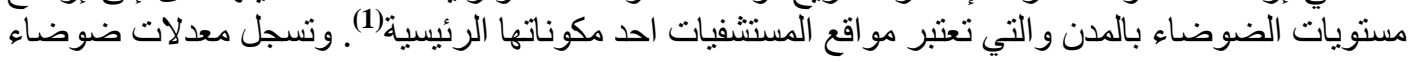


صالح بوسف الفرد وآخرون - المعالجات المعدارية لمواقع المستشفيات للحد من تأثبر الضوضاء الخارجية

الطرق بمصر قر اءات مرتفعة تجاوزت المعدلات المسموح بها الأمر الذي أثر سلباً على مختلف أنشطة الحياة

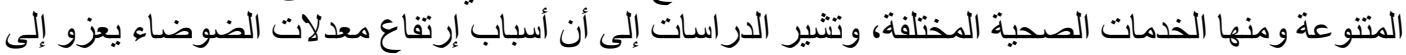

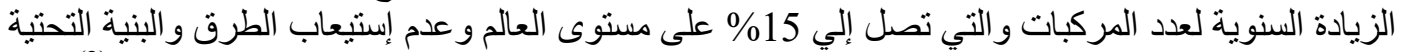

لهذه الزيادة بالإضافة إلي عدم صيانة المركبات وإستخدام مركبات قديمة وخاصة المبل المركبات الثقيلة منها.

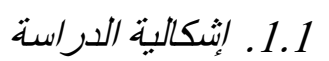

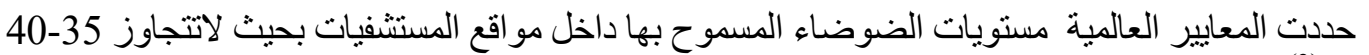

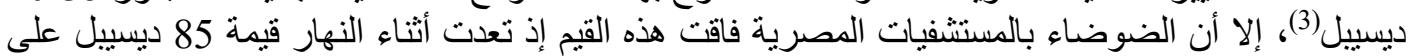

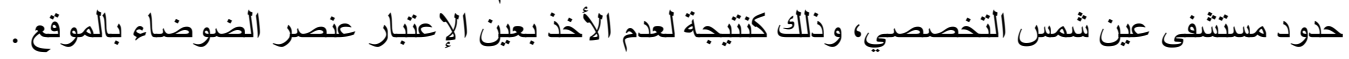

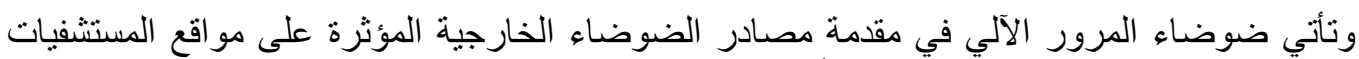

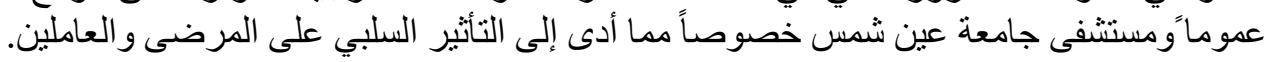

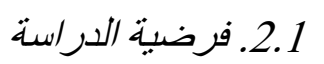

تعتمد الدر اسة فرضية أن خفض الضوضاء النداء داخل مو اقع المستشفيات يمكن تحقيقه بعدة إجر اءات معمارية لحماية مباني المستشفيات من الضوضية ان خضوضاء الضوض

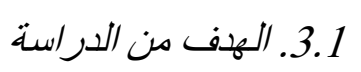

تهدف الدر اسة إلى وضع أسس و اشتر اطات للمعالجات المعمارية يمكن تطبيقها لحماية مو اقع المستشفيات المزمع تتفيذها من الضوضاء الخارجية لمدة طويلة.

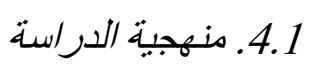

لتحقيق أهداف الدر اسة وإختبار الفرضية السابقة يتم إتباع المنهج التحليلي الإستقر ائي للمر اجع و البيانات

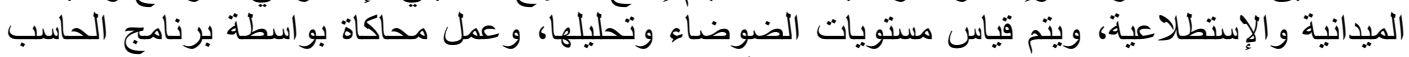

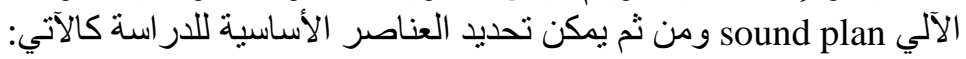

ـ معايير ومستويات الضوضاء بالمسنشفيات.

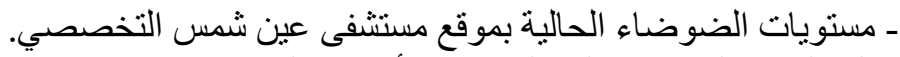
ـ المعالجات المعمارية المختلفة وتقيم تأثثير ها بالموقع.

\section{2. معاييز ومستويات الضوضاء بالمستشفيات}

قامت العديد من المنظمات و الهيئات المختصة بدراسة ووضع المعابير والمحددات اللازمة لمستويات

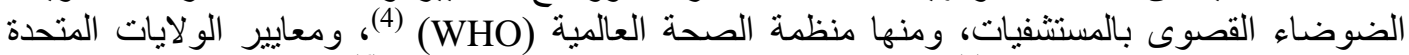

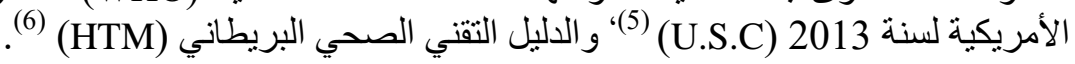
1.2. المعابير المصرية لمستوبات الضوضاء بالمستشفيات

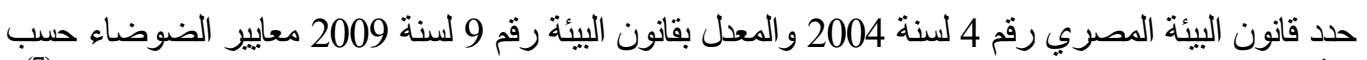

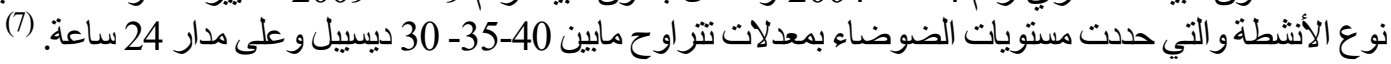
كما حدد دليل المعايير التصميمية للمستشفيات والمنشئات الصحية المصري وهو أول دليل عربي بضع

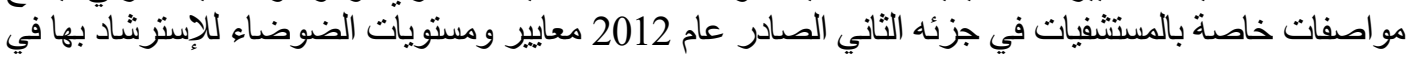

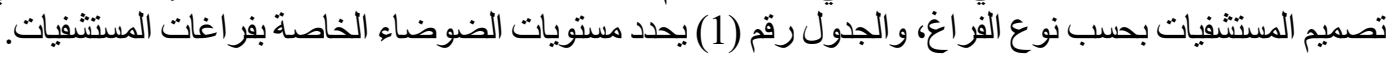




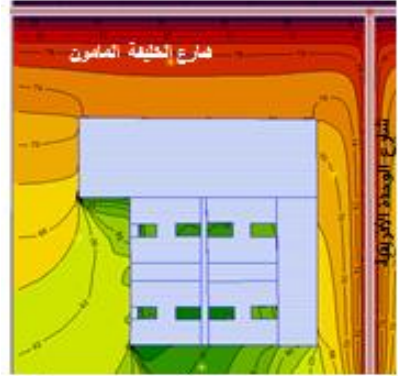

شكل رقم (2): المسقط الأفقي للموقع مبيناً عليه قيم الضوضناء

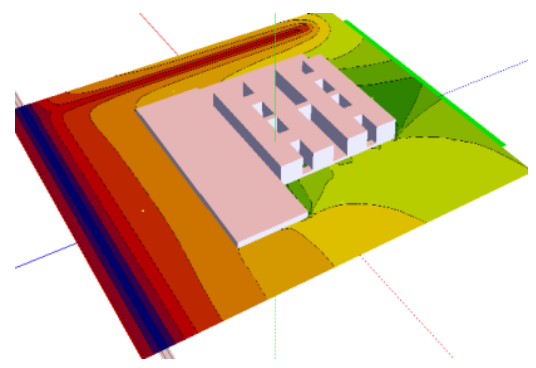

شكل رقم (1): منظور لموقع المستشفى

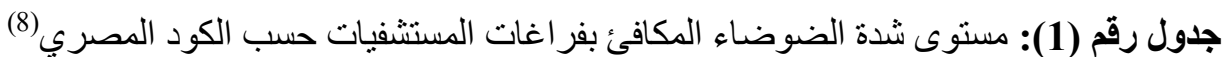

\begin{tabular}{|c|c|}
\hline Leq (dB(A)) مستوى شدة الضوضاء المكافئ & نوع الفراغ \\
\hline $40-30$ & غرفة خاصة \\
\hline $45-35$ & غرفة عمليات \\
\hline $40-35$ & غرف المرضى \\
\hline $50-40$ & معامل ،ممرات \\
\hline $50-40$ & الإستر احات و أماكن الإنتظار \\
\hline $55-45$ & دور ات المياه \\
\hline
\end{tabular}

2.2. المعابير الأمريكبة لمستويات الضوضاء للمستشفيات وتصنبفها

في دراسة أعدها خبر اء ومختصين في مجال الصوتيات بالمستشفيات بالمجلس الأعلى للمواصفات الإتهات الفنية

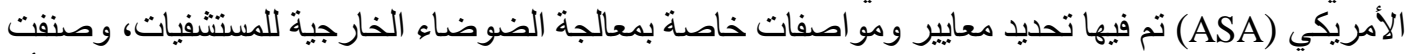

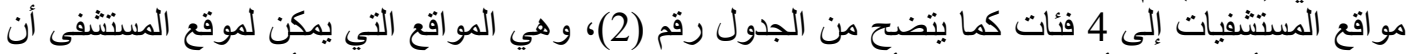

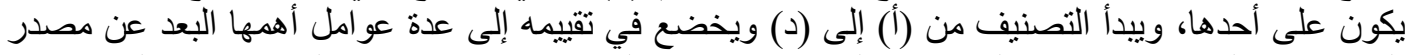

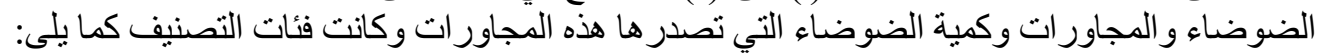

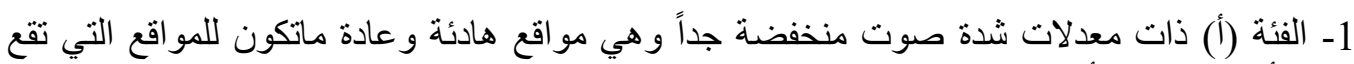

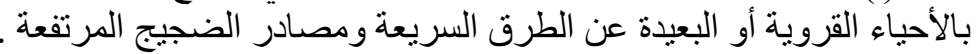

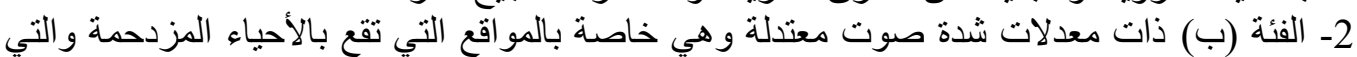

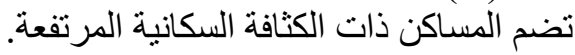

3- الفئة (ج) ذات معدلات شدة صوت درتفعة وهي خاصة بالمو اقع التي تقع في الأحياء التجارية الحضارية.

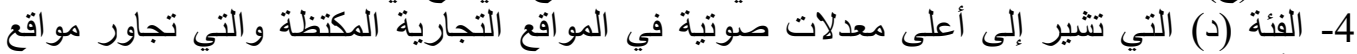

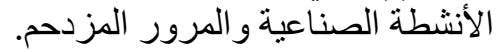

جدول رقم (2): تصنيف موقع المسنشفى بالنسبة للضوضاء الخارجية المحيط(9)

\begin{tabular}{|c|c|c|c|c|}
\hline د & ج & ب & 1 & التصنيف الرئيسي لموقع الضوضاء الخارجية \\
\hline مرتفع جداً & مرتفع & معتدل & منخفض & الوصف العام \\
\hline $75<$ & $75-70$ & $70-65$ & $65>$ & معدل مستوى الضوضـاء بالليل و النهار (dBA)(Ldn) \\
\hline $85<$ & $85-80$ & $80-75$ & $75>$ & الحد الأقصى المستمر لمستوى الضوضاء (dBA)(L10) \\
\hline $60>$ & $250-60$ & $1000-250$ & 1000 & بعد الموقع عن الطرق السريعة المحيطة بالمتر \\
\hline $1800>$ & $3500-1800$ & $7000-3500$ & $7000>$ & بعد الموقع عن مسار ات حركة الطائرات بالمتر \\
\hline $100>$ & $500-100$ & $1500-500$ & 1500 & بعد الموقع عن مسار ات سكة الحديد بالمتر \\
\hline 50 & 45 & 40 & 35 & مستوى الضوضاء على الحدود الخارجية للمبنى (dBA)(Ldn) \\
\hline
\end{tabular}


صالح بوسف الفرد وآخرون - المعالجات المعدارية لمواقع المستشفيات للحد من تأثبر الضوضاء الخارجية

\section{3. مستويات الضوضاء بأحد مواقع المستشفيات المصرية}

يحتبر مستشفى عين شمس التخصصي من مستشفيات جامعة عين شمس الهامة و الكبرى بمنطقة العباسية بالقاهرة، وقد إستمد

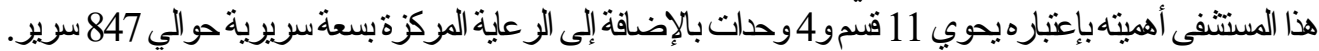
وتتكون كتلة مبنى المستشفى من الطابق الأرضي الذي يحوي أقسام مبنى العيادات الخارجية المعامل و العمليات

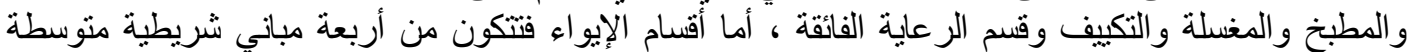
الإرتفاع هي ( أـ ب- حـ د) وينكون كل مبنى من 3 طو ابق الأول يحوي أقسام الإيو اء المختلفة بالمستشفى (10).

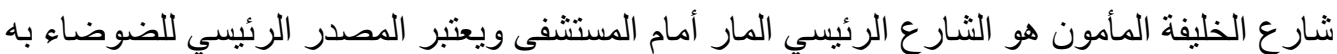

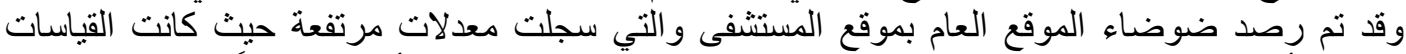

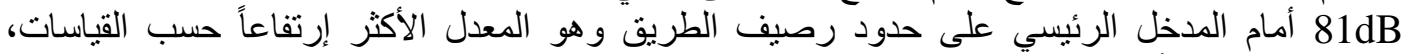

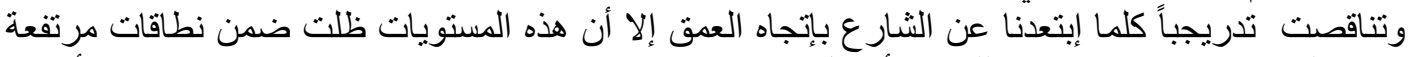

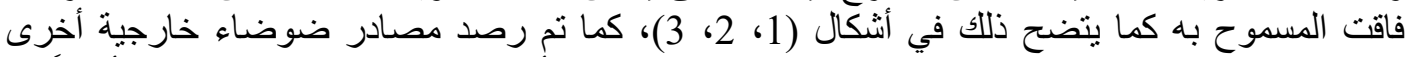

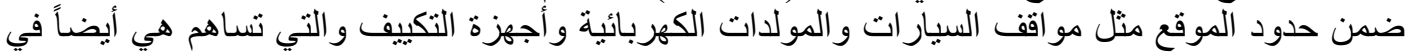

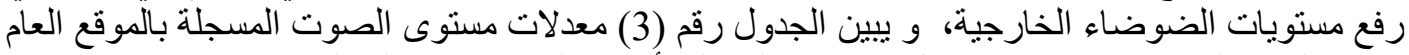

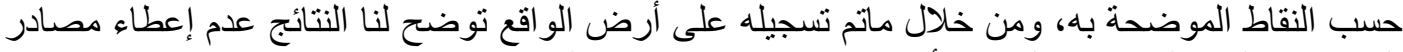

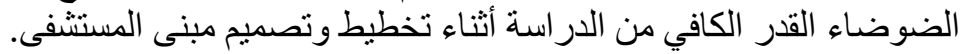

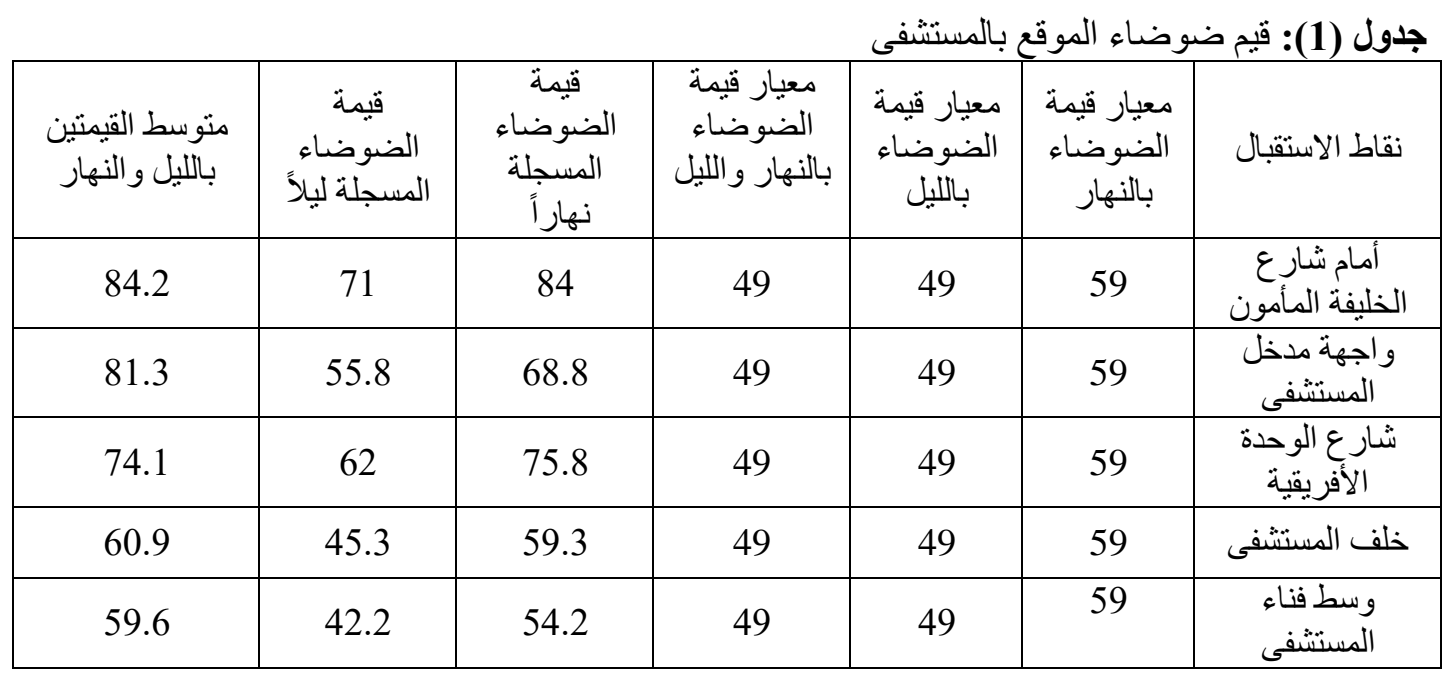

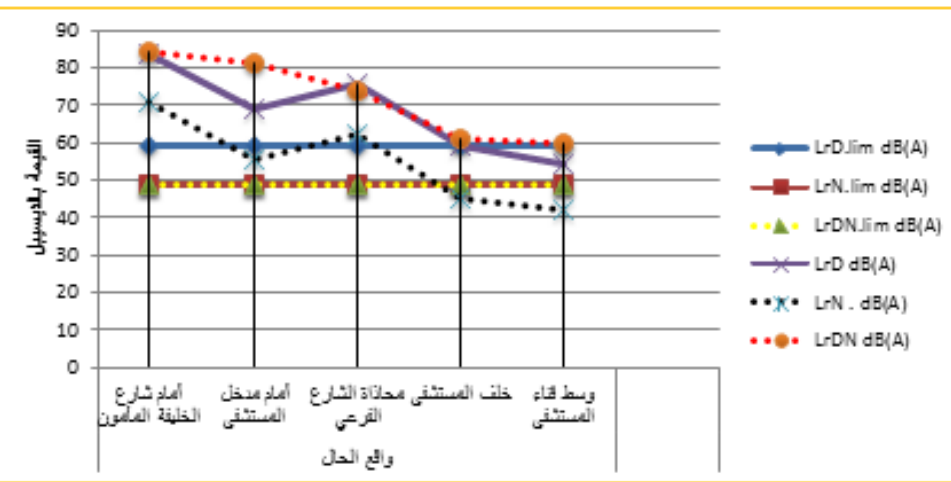

شكل رقم(3): قيم معدلات الضوضاء المختلفة المسجلة بالموقع العام 
JES, Assiut University, Faculty of Engineering, Vol. 44, No. 3, May 2016, pp. 310 - 321

4. المعالجات المعمارية للحد من الضوضاء

$$
\text { 4.1 التوجيه بالنسبة لهصدر الضوضاء }
$$

يتأثر المبنى بعدة محددات بيئية بنبخي الأخذ بها عند دراسة وتصميم المستشفيات مثل الإضاءة الطبيعية

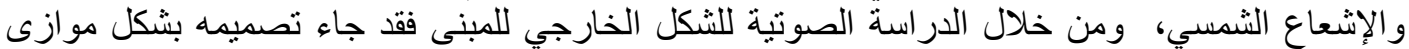

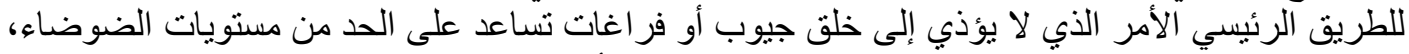

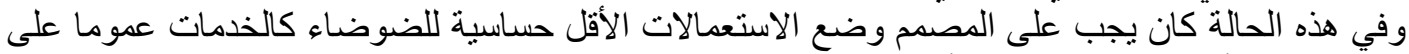
هذا الجانب، أما تصميم الطابق الأرضي الموازي لإني للطريق فهو يحتوى على المدخل الرئيسي والاستقبال

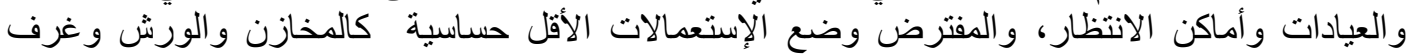

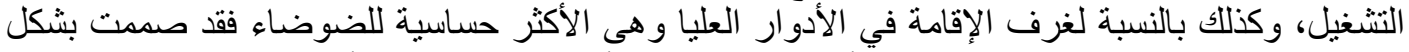

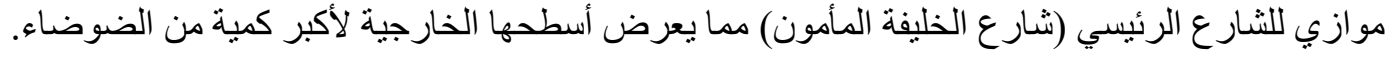

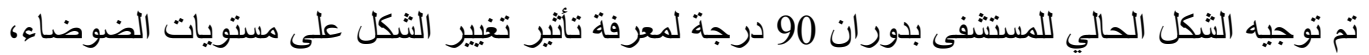

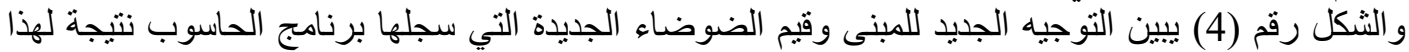
التغيير، حيث يبين الجدول رقم (4) القيم الجديدة للضوضناء المسجلة بالموقع ومقارنته بعد إعادة التوجيه

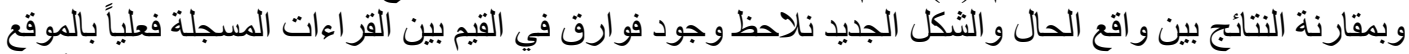
و القراءات المسجلة بعد تغيير اتجاه المبنى، حيث كانت قراءة واته واقع الحال للنقطة المقابلة لثنار ع الخليفة المأمون

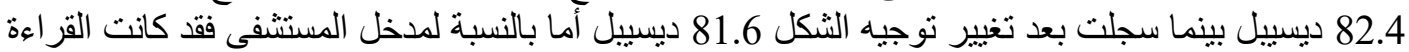

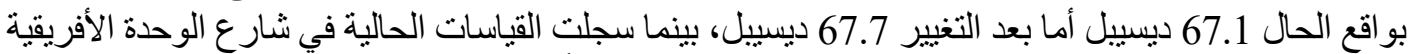

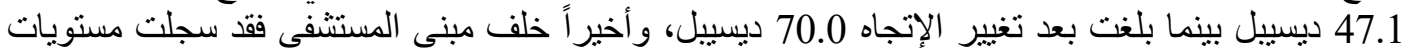

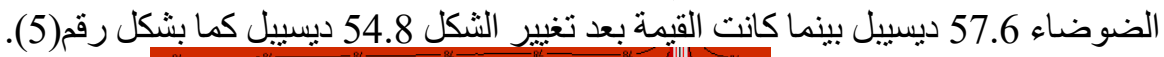

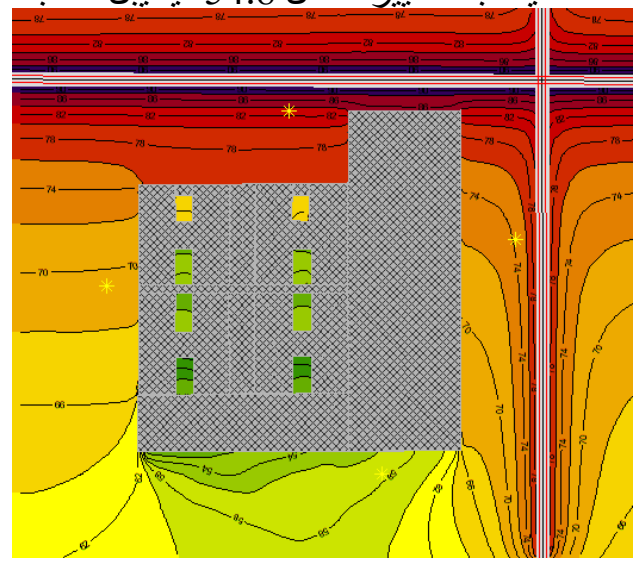

شكل رقم (4): القيم المختلفة للضوضناء بعد إعادة التوجيه

جدول (4): مقارنة بين القياسات الحالية وبعد إعادة توجيه المبنى

\begin{tabular}{|c|c|c|c|c|c|}
\hline متالليل و النهار القيمتين & 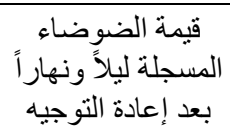 & بالنهار و اللياء قيمة & الضيوضار فيمة & الضوضاء بالنهار & نقطة الرصد \\
\hline 82.4 & 81.6 & 49 & 49 & 59 & الخليفة المأمون \\
\hline 67.1 & 67.7 & 49 & 49 & 59 & أمسام مدخل \\
\hline 74.1 & 70.9 & 49 & 49 & 59 & محاذاة الثـارع \\
\hline 57.6 & 54.8 & 49 & 49 & 59 & خلف المستشفى \\
\hline
\end{tabular}


صالح بوسف الفرد وآخرون - الدعالجات المعدارية لمواقع المستشفيات للحد من تأثبر الضوضاء الخارجية. ....

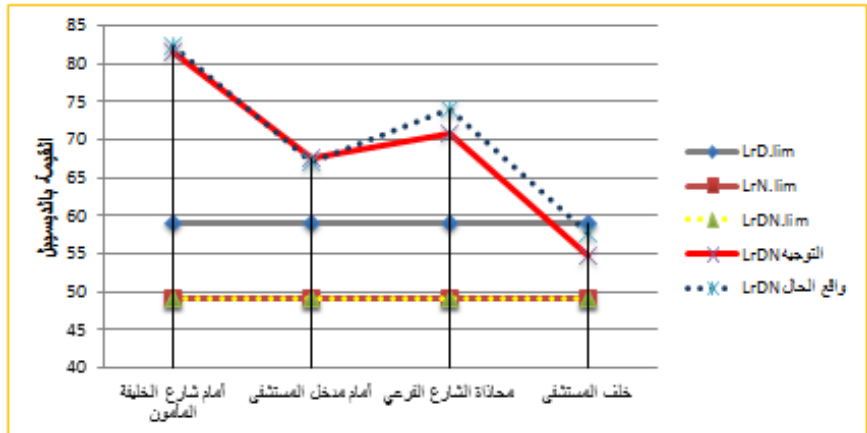

شكل رقم (5): يقارن بين قيم ضوضاء التوجيه الجديد وو اقع حال المستشفى

2.4 . تغبر شكل كثلة المبنى

يلعب تغيير شكل كتلة مبنى المستشفى دور في إمكانية خفض قيم الضوضاء إذا ما تم توظيفيه بالثنكل

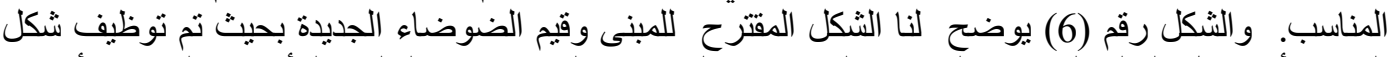

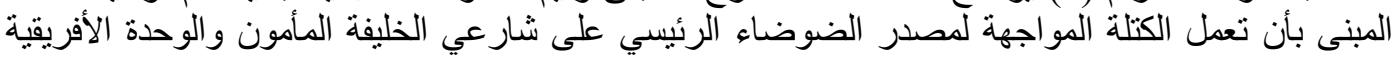

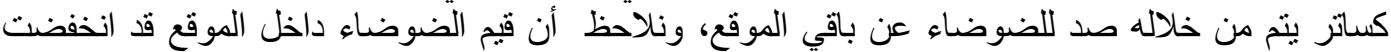

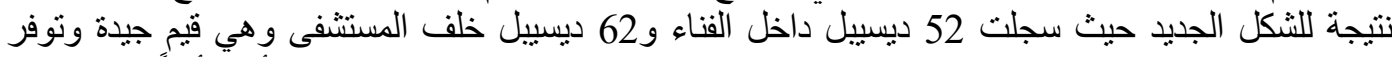

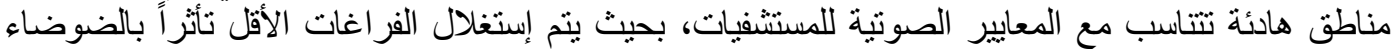

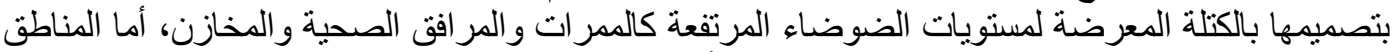

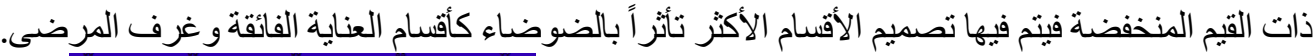
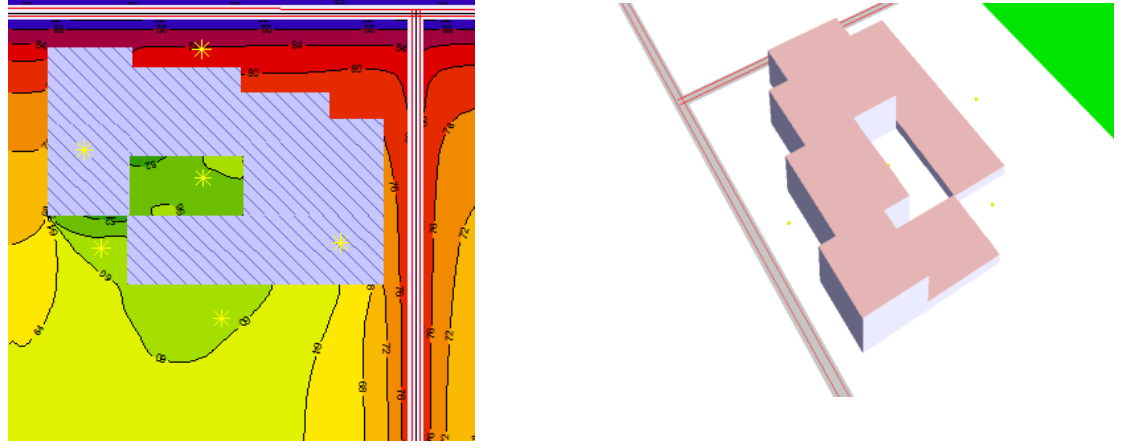

شكل رقم (6): قيم الضوضاء المختلفة المسجلة بالموقع العام بعد تغيير شكل المبنى

جدول (5): يقارن بين قيم الضوضاء الفعلية بالموقع وقيم الضوضاء بعد تغيير الثكل

\begin{tabular}{|c|c|c|c|c|c|c|c|}
\hline والقيمتين & الضوضلة المسلة & الضوضلة المسلة & معيار قيمة & الضوضار قيمة & الضوضار قبمة & نقاط الاستقبال & \\
\hline 82.4 & 71 & 84 & 49 & 49 & 59 & أمام شار ع الخليفة المأمون & \multirow{5}{*}{$\frac{\sqrt{2}}{\overline{3}}$} \\
\hline 67.1 & 55.8 & 68.8 & 49 & 49 & 59 & أمام مدخل المستشفى & \\
\hline 74.1 & 62 & 75.8 & 49 & 49 & 59 & محاذاة الشار ع الفرعي & \\
\hline 57.6 & 45.3 & 59.3 & 49 & 49 & 59 & خلف المستشفى & \\
\hline 52.6 & 42.2 & 54.2 & 49 & 49 & 59 & وسط فناء المستشفى & \\
\hline
\end{tabular}


JES, Assiut University, Faculty of Engineering, Vol. 44, No. 3, May 2016, pp. 310 - 321

\begin{tabular}{|c|c|c|c|c|c|c|c|}
\hline والقيمتين & الضوضلة المسلة & الضوضلاء & مبال الضوضاء قيمة & الضوضار قبمة & مبيار قيمة & نقاط الاستقبال & \\
\hline 81.9 & 70.6 & 83.3 & 49 & 49 & 59 & أمام شار ع الخليفة المأمون & \multirow{5}{*}{$y$} \\
\hline 56.2 & 44.7 & 67.7 & 49 & 49 & 59 & أمام مدخل المستشفى & \\
\hline 57.4 & 45.3 & 59.1 & 49 & 49 & 59 & محاذاة الثار ع الفرعي & \\
\hline 53.6 & 42 & 55.2 & 49 & 49 & 59 & خلف المستشفى & \\
\hline 48.4 & 40.2 & 51.3 & 49 & 49 & 59 & وسط فناء المستشفى & \\
\hline
\end{tabular}

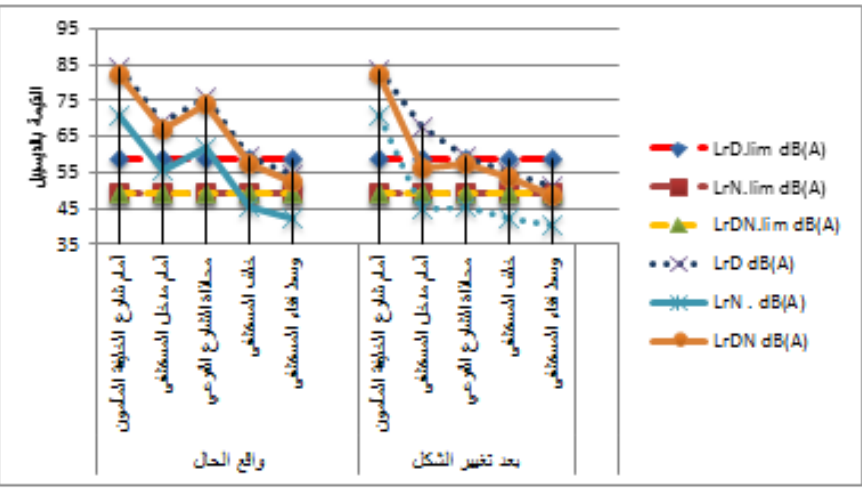

شكل رقم (7): مقارنة بين قيم الضوضاء الفعلية بالموقع وقيم الضوضاء بعد تغيير الشكل

3.4 الأشجار و المسطحات الخضراء

تلعب الأشجار دور هام في خفض الضوضاء بأ بالمسنشفيات وتتعدد فو ائد إستخدامها في الموقع من الناحية البيئية

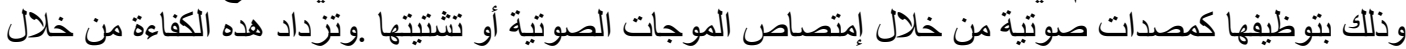

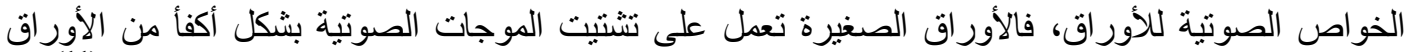

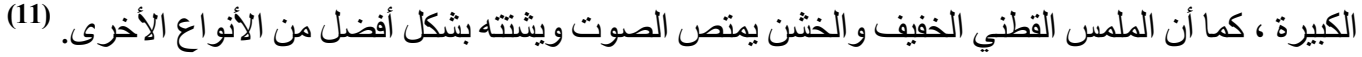

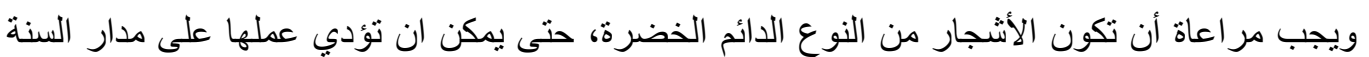

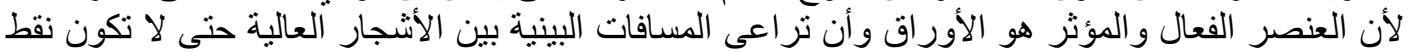

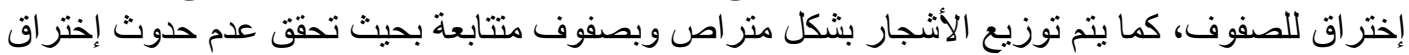

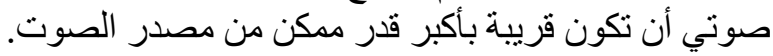

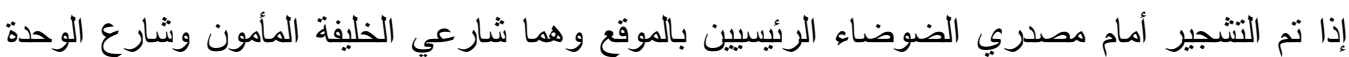

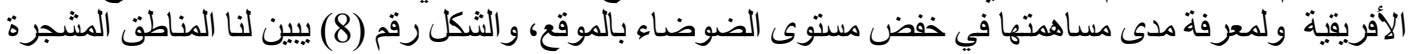

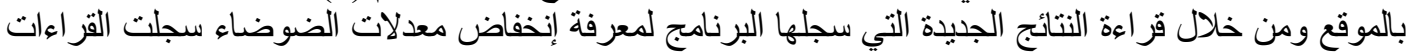

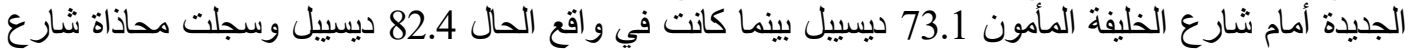

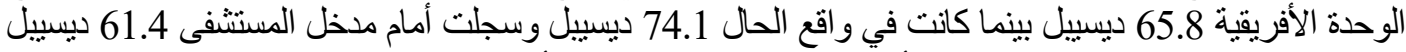

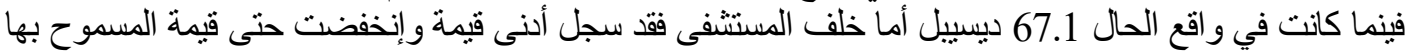

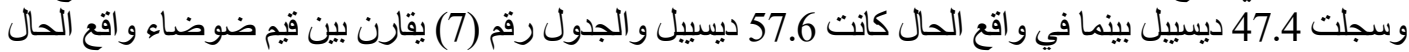

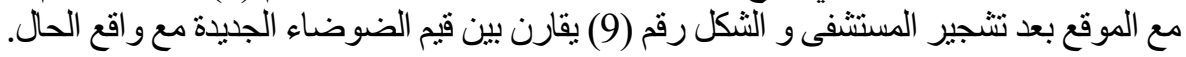



صالح بوسف الفرد وآخرون - المعالجات المعدارية لمواقع المستشفيات للحد من تأثبر الضوضاء الخارجية
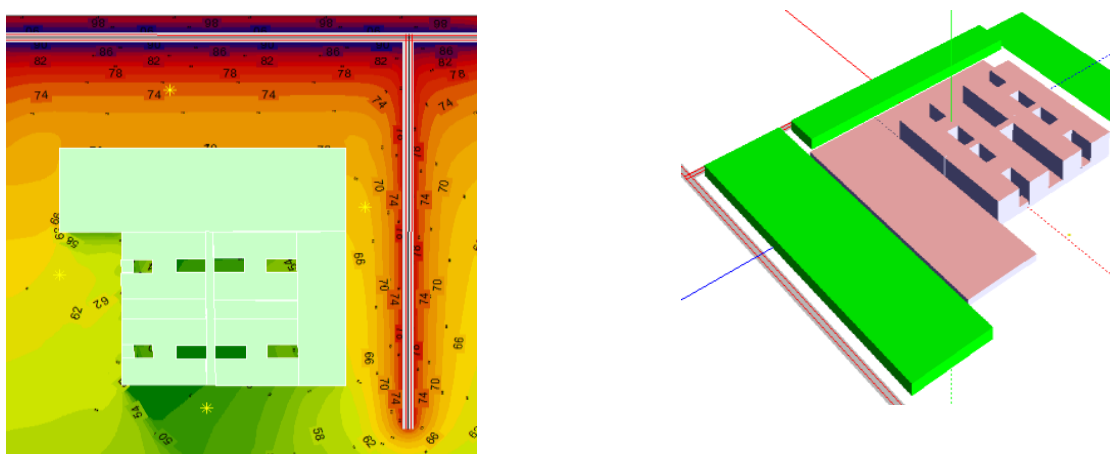

شكل رقم (8): خريطة كونتورية توضح كتلة المستشفى ومنطقة التشجير

جدول رقم (7): مقارنة يقارن بين قيم الضوضاء الفعلية بالموقع و القيم بعد مقتر ح المناطق الخضر اء

\begin{tabular}{|c|c|c|c|c|c|c|c|}
\hline متو القيمتين & الضوضة المسلةً & الضوضاء & الضوضار فياء & معيار قيمة & بالضوضار قيمة & نقاط الاستقبال & \\
\hline 84.2 & 71 & 84 & 49 & 49 & 59 & أمام شار ع الخليفة المأمون & \multirow{5}{*}{$\begin{array}{l}\frac{9}{2} \\
\overline{5}\end{array}$} \\
\hline 81.3 & 55.8 & 68.8 & 49 & 49 & 59 & أمام مدخل المستشفى & \\
\hline 74.1 & 62 & 75.8 & 49 & 49 & 59 & محاذاة الثار ع الفرعي & \\
\hline 60.9 & 45.3 & 59.3 & 49 & 49 & 59 & خلف المستشفى & \\
\hline 59.6 & 42.2 & 54.2 & 49 & 49 & 59 & وسط فناء المستشفى & \\
\hline 72.7 & 70.6 & 83.3 & 49 & 49 & 59 & أمام شار ع الخليفة المأمون & \multirow{5}{*}{ 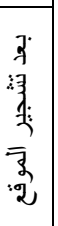 } \\
\hline 56.2 & 44.7 & 67.7 & 49 & 49 & 59 & أمام مدخل المستشفى & \\
\hline 57.4 & 45.3 & 59.1 & 49 & 49 & 59 & محاذاة الثار ع الفرعي & \\
\hline 53.6 & 42 & 55.2 & 49 & 49 & 59 & خلف المستشفى & \\
\hline 48.4 & 40.2 & 51.3 & 49 & 49 & 59 & وسط فناء المستشفح & \\
\hline
\end{tabular}

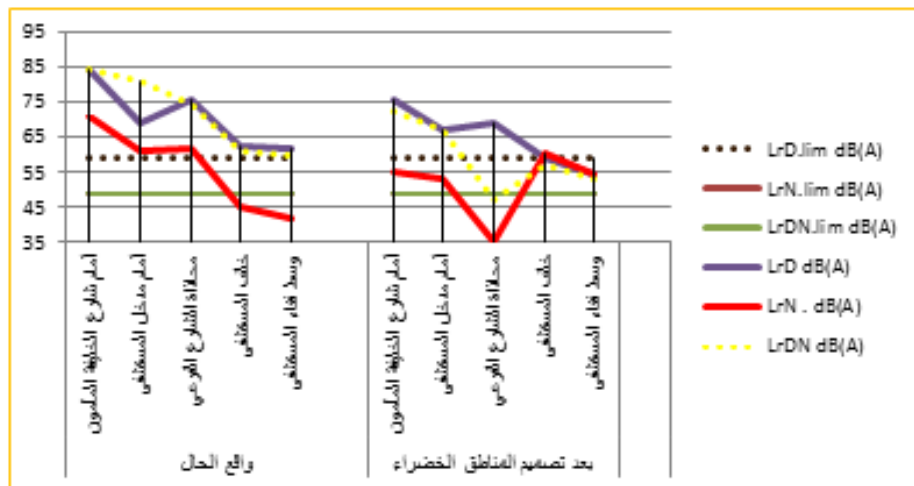

شكل رقم(9): قيم الضوضاء المختلفة المسجلة بالموقع العام بعد تطبيق المناطق الخضر اء

$$
\text { 4.4. تصدبم الأســــورل }
$$

تم مراعاة بعض الأساسيات المهمة عند إستخدام الأسوار كحاجز للصوت في موقع المستشفى بحيث تم

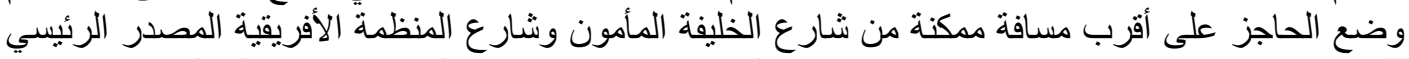

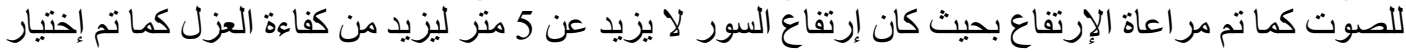

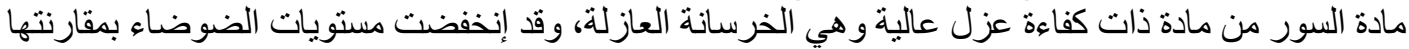
بالمستويات الحالية حيث سجلت القراءات الجديدة أمام شارع الخليفة المأمون 65 ديسييل بينما كانت في واقع إنع 
JES, Assiut University, Faculty of Engineering, Vol. 44, No. 3, May 2016, pp. 310 - 321

الحال 82.4 ديسييل وسجلت محاذاة شارع الوحدة الأفريقية 59.1 ديسييل بينما كانت في واقع الحال 74.1 دئل ديسيبل وسجلت أمام مدخل المستشفى 52.7 ديسييل فينما كانت في واقع الحال 67.1 ديسييل، أما خلف المستشفى فقد سجل أدنى قيمة و إنخفضت حتى قيمة المسموح بها وسجلت 41.3 ديسيبل بينما في واقع الحال

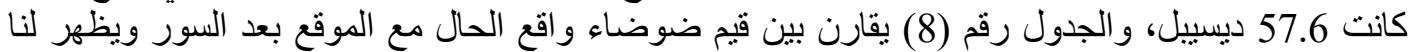

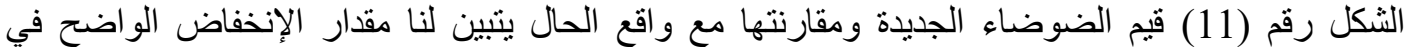

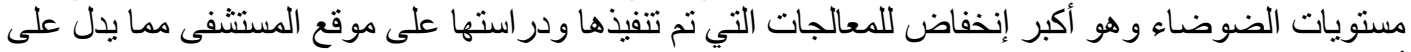
أفضلية ونجاح هذا النوع من المعالجات في خفض قيمة الضوضاء بموقع المستشفى كما في الثكل رقم (10).
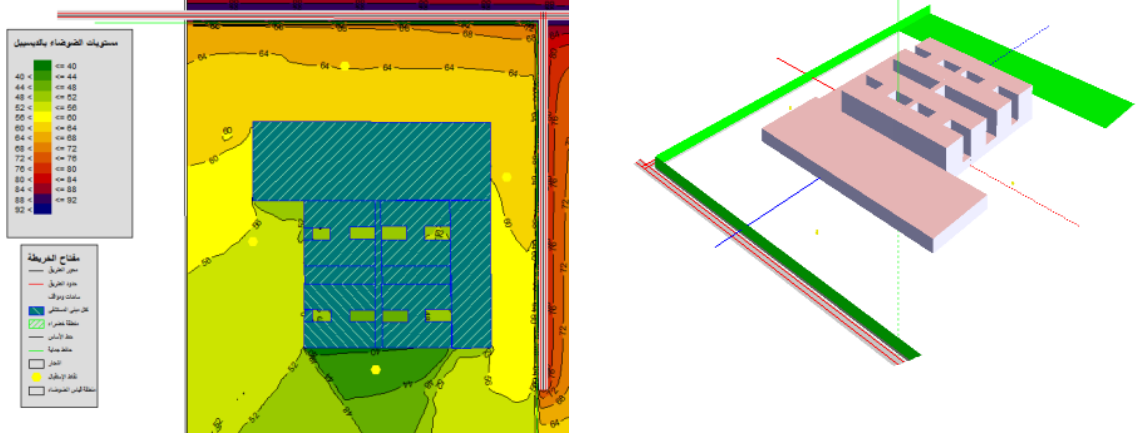

شكل رقم (10): خريطة كونتورية للضوضاء تبين كتلة المستشفى بعد تصميم السور

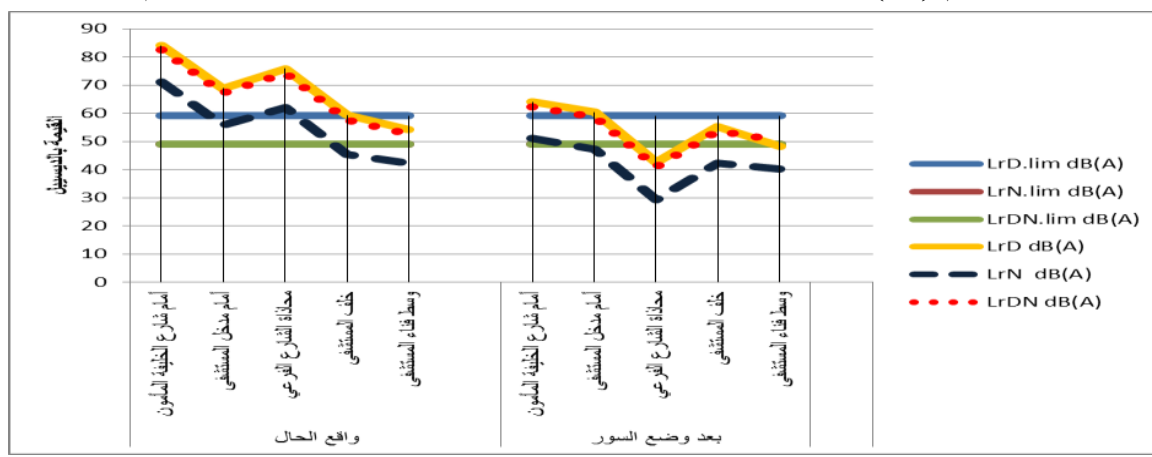

شكل رقم (11): يقارن بين قيم الضوضاء الفعلية و القيم بعد تصميم السور

جدول رقم (8): بقارن بين قيم الضوضاء بالموقع بو اقع الحال وبعد تصميم السور

\begin{tabular}{|c|c|c|c|c|c|c|}
\hline بالليل و القيمتين & لضيوةًاء & الضوضاء & بالضهار و الضياء & الضوضار قيمة & الضيوضاء قياء & نقاط الاستقبال \\
\hline 82.4 & 71 & 84 & 49 & 49 & 59 & أمام شار ع الخليفة المأمون \\
\hline 67.1 & 55.8 & 68.8 & 49 & 49 & 59 & أمام مدخل المستشفى \\
\hline 74.1 & 62 & 75.8 & 49 & 49 & 59 & محاذاة الثار ع الفرعي \\
\hline 57.6 & 45.3 & 59.3 & 49 & 49 & 59 & خلف المستشفى \\
\hline 52.6 & 42.2 & 54.2 & 49 & 49 & 59 & وسط فناء المستشفى \\
\hline 62.3 & 51 & 64 & 49 & 49 & 59 & أمام شار ع الخليفة المأمون \\
\hline 58.7 & 47.2 & 60.4 & 49 & 49 & 59 & أمام مدخل المستشفى \\
\hline 40.8 & 29.3 & 42.5 & 49 & 49 & 59 & محاذاة الثار ع الفرعي \\
\hline 53.7 & 42.3 & 55.3 & 49 & 49 & 59 & خلف المستشفى \\
\hline 50.4 & 40.1 & 48.2 & 49 & 49 & 59 & وسط فناء المستشفى \\
\hline
\end{tabular}


تتعرض هذه الورقة البحثية إلى معالجات الموقع العام و إمكانية تخفيض الضوضاء من خلال مجمو عة من

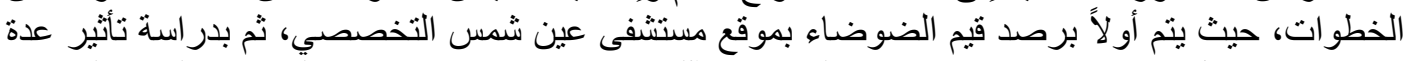

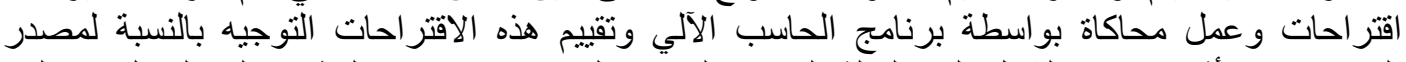

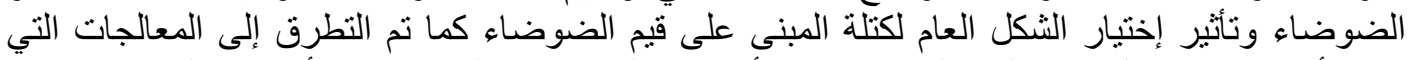

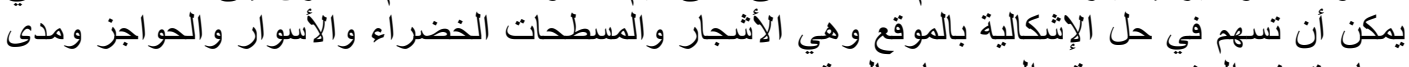

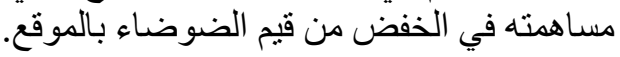

ومن خلال الدر اسة على مستشفى عين شمس التخصصي المختارة والنتائج التي تم التوصل إليها يمكن الوصول إلى النتائج التالية:

- مستويات الضوضاء الخارجية بالمستشفيات المصرية التي تم در استها سجلت إرتفاعاً ملحوظاً

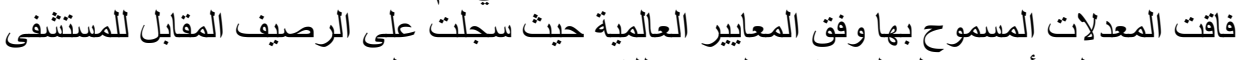

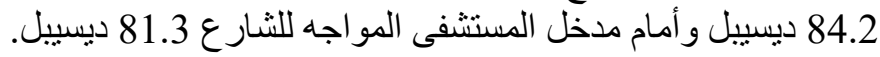
- يفتقد التخطيط العام للموقع الأخذ بعين الإعتبار عنصر الضوضاء كأحد العناصر الهامة المؤثرة على كتلة المستشفى.

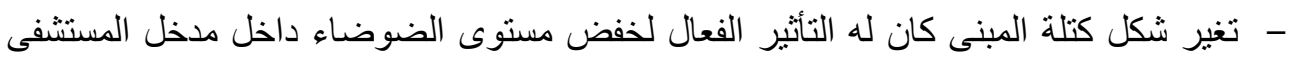

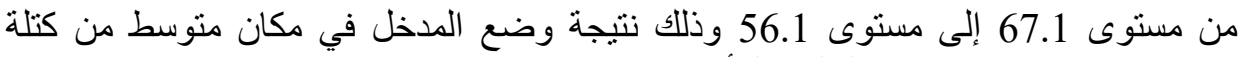
مندرجة ومنباعدة عن شار ع الخليفة المأمون.

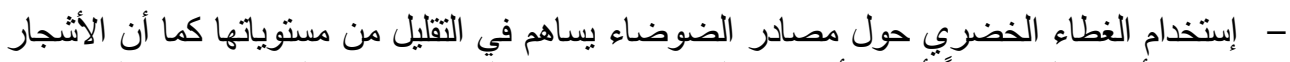

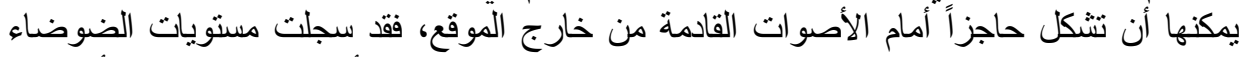

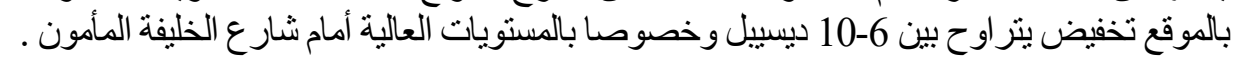
- - الحواجز و الأسوار توفر الحماية ويمكنها الحد من قيم مستويات الضوضاء المؤثرة على الموقع وهي

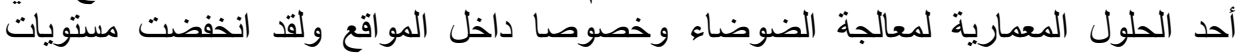

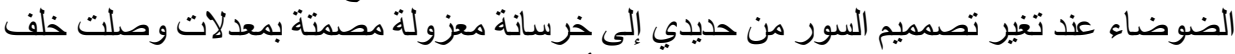

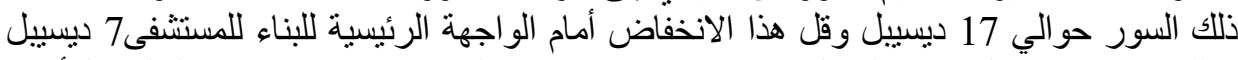

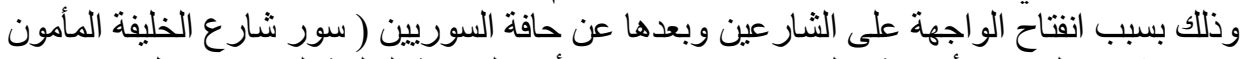

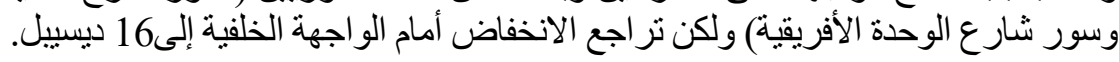

\section{6 التوصيات}

للوصول إلى أفضل النتائج للحد من ضوضاء الموقع علينا إتباع الآتي:

- ير اعى اختيار مو اقع المسنتفيات أن تكون بعيدة عن الطرق السريعة و المطار ات و السكك الحديدية .

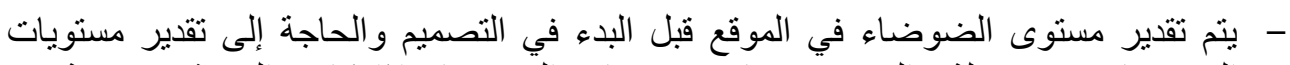

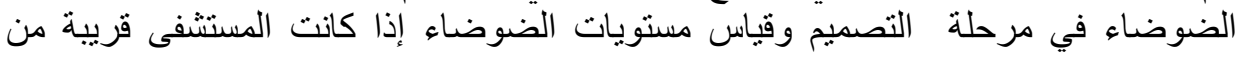

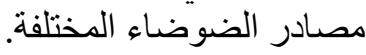

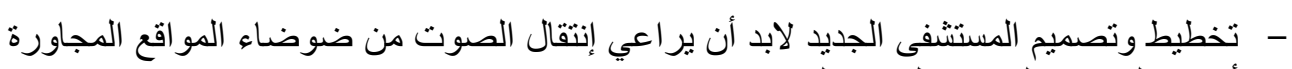

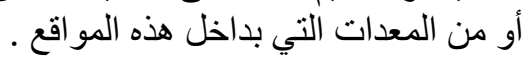

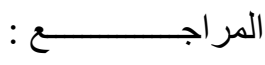

[1] العز ازي، محمد عبدالو هاب " تأثير الضوضاء على تحديد استعمالات الأراضي بالمدن المصرية" رسالة دكتو ارة، جامعة أسيوط، معد 2005. 
JES, Assiut University, Faculty of Engineering, Vol. 44, No. 3, May 2016, pp. 310 - 321

[2] Byers JF ،Waugh WR\& Lowman ‘LB "Sound Level Exposure of High-risk Infants in Different Environmental Conditions" Neonatal Intensive Care Units، Critical Care Nurse، 2006.

[3] Lewis S and RL Cardinal" Noise in Hospital" (An acoustical study of noises affecting the Patient) 1985 .

[4] Dietrich Schwela "World Health Organization Guidelines on Community Noise" TRB Session 391 Setting an Agenda for Transportation Noise Management Policies in the United States، Washington DC، USA، 2001.

[5] United States Code Edition Title 42 "The Public Health and Welfare Chapter" 852013.

[6] Department of Health "Health Technical Memorandum (HTM) "2012.

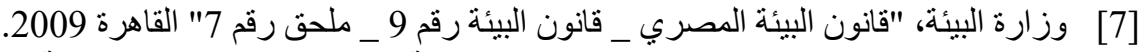

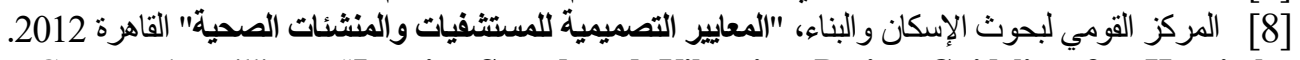

[9] Cavanaugh William J"Interim Sound and Vibration Design Guidelines for Hospital and Healthcare Facilities" Public Draft 1،p10، AIA Healthcare Guideline Revision Committee 2006.

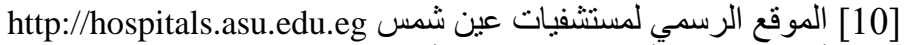

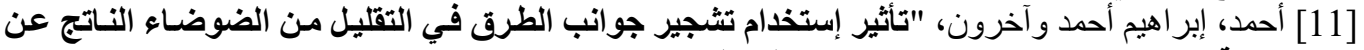

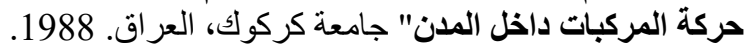


صالح بيوسف الفرد وآخرون - المعالجات المعدارية لمواقع المستشفيات للحد من تأثبر الضوضاء الخار جية ..................

\title{
ARCHITECTURAL TREATMENT \\ OF THE HOSPITALS SITES TO LIMITING OUTDOOR NOISE, EINSHAMS HOSPITAL, CAIRO, EGYPT
}

\begin{abstract}
To become the hospital Building project is succeeded، it should be protected it from exterior noise the protection process include acoustics studies، hospital site selecting is suitable to noise allowance limited، and studying a noise element as one of factors which effect in hospitals planning and design. The study aims to find a means can reduce the noise at Einshams hospital site (located at one of the most crowded streets in Cairo is named Elkaliafa elmamoun street. Systematically it is followed to reduce $t$ neglected effects on the patients ، employers، and staff members.

To reach the aims، it can follow as:1- data collect and noise levels measure by S.L.M. 2- the measurements analyse to assess noise effect at the site. 3-the suitable solutions and treatments suggest to reduce the noise levels' and then they is examined by software program. They are as: direction the form to the noise source- choosing and changing the form- buffer zone and distanceconstruction a noise wall.
\end{abstract}

The paper ends with a number of planning and architectural criteria which should be taken into account to reduce the influence of outdoor noise at the hospital site.

Keywords: Hospital site- outdoor noise - noise level-traffic noise 